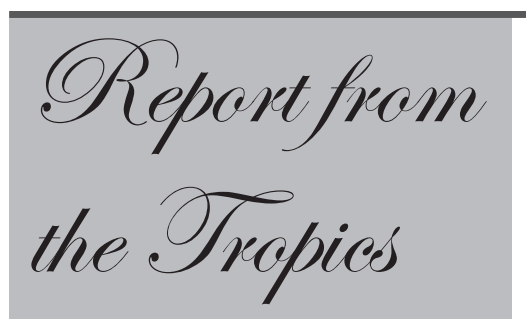

\title{
Feasibility and Safety Assessment of Home Based Gastrostomy Tube Feed - A Tertiary Care Centre Experience
}

\author{
Avinash Balekuduru ${ }^{1}$, Abhinav Kumar ${ }^{1}$, \\ Amit Kumar Dutta ${ }^{2}$, Hema Aravind ${ }^{3}$, Satyaprakash \\ Bonthala Subbaraj ${ }^{1}$
}

${ }^{1}$ Department of Gastroenterology,

ABSTRACT

Ramaiah Memorial Hospitals,

Bangalore, Karnataka, India

${ }^{2}$ Department of Gastroenterology,

Christian Medical College and Hospitals,

Vellore, Tamilnadu, India

${ }^{3}$ Department of Clinical Nutrition,

Ramaiah Memorial Hospitals,

Bangalore, Karnataka, India

Corresponding Author:

Dr Avinash Balekuduru

Email: avinashbalekuduru@gmail.com

Background and Aim: The type of percutaneous endoscopic gastrostomy (PEG) tube feed may result in improved survival or increased mortality. The aim of the present study was to assess the safety of a novel home based PEG tube feed preparation.

Method: Subjects who were on home based PEG feed for enteral nutrition at our center between September 2016 and August 2017 were analyzed retrospectively. The indication for PEG, clinical and feeding related adverse effects were recorded on uniform structured data forms.

Results: One hundred and five PEG patients (76\% men)with mean age of $50.8+10$ years were enrolled. Road traffic accidents in $63(60 \%)$ and peristomal infection in $19(18 \%)$ comprised the major indication and adverse effects of PEG placement respectively. There was no mortality during the study period.

Conclusions: Home based gastrostomy tube feed is safe and can be a cheaper alternative in low cost setting.

KEYWORDS: Gastrostomy tube feed, Percutaneous endoscopic gastrostomy.

\section{Introduction}

The maintenance of appropriate nutrition in patients with chronic illness is well recognized as a fundamental part of standard medical care. Malnourished patients have poorer clinical outcomes and more complications than well-nourished patients. ${ }^{1}$ Enteral as compared to parentral nutrition is simpler, safer, economical and maintains mucosal barrier function. Percutaneous endoscopic gastrostomy (PEG) placementis a common practice in patients requiring enteral feeding for more than four to five weeks. ${ }^{2}$ PEG feeds can be either commercially available or homemade. Commercially available formula feeds differ in osmolarity, caloric density, amount of protein , calorie, vitamin, mineral and electrolyte content. But they come with a high cost. Due to limited resources and 
non-availability of low cost PEG feeds, poor nourishment can be anticipated. Semisolid feeds are developed as an alternative to liquid feeds to reduce feeding related adverse events. ${ }^{3}$ The aim of the current study is to assess the safety of a home based PEG feeding formula from our center. The safety and efficacy of feeding formula is assessed by clinical outcome and feeding related adverse events.

\section{Patients and Methods}

Subjects who underwent endoscopic PEG (24 Fr WilsonCook Corporation, Winston-Salem, North Carolina, USA) placement for enteral nutrition at our center between September 2016 and August 2017 were included retrospectively. Subjects who did not give consent for the home based formula were excluded from the study. A novel home prepared feeding formula as given in table $\mathbf{1}$ was advised for PEG feeds.

\section{Feeding Protocol and Formulation}

The calorie content was designated by the nutritionist depending on whether they are taking orally or not. All kinds of seasonal vegetables with low fiber are chosen to avoid blocking the tube (figure 1). The seeds of spine gourd and bitter gourd are hard and to be avoided. One scoop of moong dal and three scoops of brown rice are added along with 1 medium onion,garlic, salt and fried boneless fish 50-100 gm or two egg white. The base can be modified as red rice or brown rice or ragi or broken wheat as required. Pressure cooked with adequate water till 4-5 whistles, blended till paste is noted (figure 2,3). Feeding is done with patient in semi reclined position at 45 degree to avoid aspiration. Supine positioning and suctioning is avoided for 1 hour post feed to avoid aspiration. Asepto syringe- Ramsons- Agra (1990) is fixed at the end of the PEG tube and the blended feed is squeezed using the balloon. The feeding volume varies with the condition of the patient. Once feeding is completed, syringe is washed

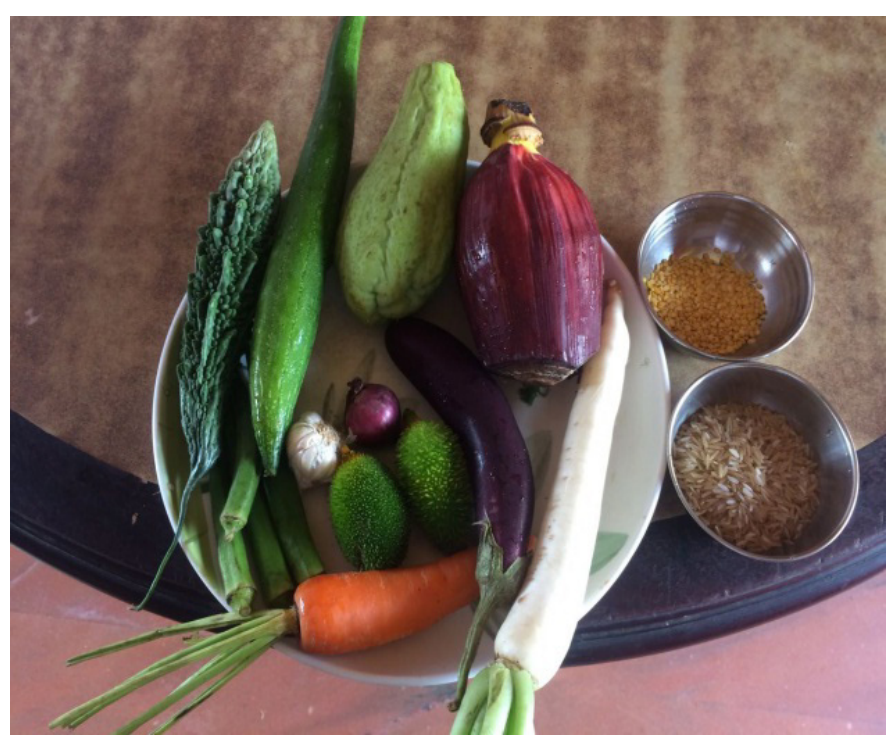

Figure 1: Showing collection of vegetables.

Table 1: Home based modified IndraPEG feed formula.

\begin{tabular}{l|l|l|l|l|l|l} 
& Major Nutrient & $\begin{array}{l}\text { Quantity } \\
\text { in grams }\end{array}$ & $\begin{array}{l}\text { Energy } \\
\text { in Kcal }\end{array}$ & $\begin{array}{l}\text { Carbohydrate } \\
\text { in grams }\end{array}$ & $\begin{array}{l}\text { Protein } \\
\text { in grams }\end{array}$ & $\begin{array}{l}\text { Fat in } \\
\text { grams }\end{array}$ \\
\hline Moong Dal & Protein & 20 & 70 & 12 & 5 & 0.2 \\
\hline Brown Rice & Fiber, Magnesium, Phosphorus & 15 & 17 & 6.4 & 0.3 & 0.1 \\
\hline Carrot & Vitamin A, K, Potassium & 100 & 48 & 10.6 & 0.9 & 0.2 \\
\hline Karela & Vitamin C, Magnesium, Potassium & 120 & 30 & 5 & 1.9 & 0.2 \\
\hline Brinjal & Potassium, Fiber & 50 & 12 & 2 & 0.7 & 0.1 \\
\hline Onion & Vit C & 100 & 50 & 11.1 & 1.2 & 0.1 \\
\hline Bhindi & Vitamin A, C,K, Folic acid & 40 & 14 & 2.5 & 0.7 & 0.08 \\
\hline Garlic & Selenium, Vitamin C & 30 & 36 & 7.4 & 1.5 & 0.02 \\
\hline Cucumber & Vitamin K, Molybdenum & 150 & 28 & 5.5 & 0.8 & 0.2 \\
\hline Chayote & Vitamin A,B1,B2 & 200 & 54 & 11.4 & 1.4 & 0.2 \\
\hline
\end{tabular}


and PEG tube is flushed with $60-100 \mathrm{ml}$ of water to ensure clean tube. Medication can be continued after the feeding and before removing from the tube. The syringe is washed and using baby feeding bottle sterilizer sterilised for the next feed.

The indication for PEG, clinical, investigation details and final etiological diagnosis were recorded on uniform structured data forms. On follow up feeding related adverse effects and outcomes were analyzed

\section{Results}

One hundred and five PEG patients (76\% men)with mean age of $50.8+10$ years were enrolled. Road traffic accidents in $63(60 \%)$ followed by carcinoma esophagus in $21(20 \%)$, stroke in $19(18 \%)$ and motor neuron disease in $2(2 \%)$ were indications for PEG in our cohort. Peristomal infection in $19(18 \%)$, aspiration pneumonia in $10(10 \%)$, tube obstruction in $9(9 \%)$ and feeding related diarrhea in $8(8 \%)$ were the adverse events observed. There was no mortality during the study period.

\section{Discussion}

PEG tube as compared to nasogastric tube (NG) is more acceptable route for enteral nutrition as the calibre is bigger and can be placed for longer periods of time. ${ }^{4}$ On NG feeds pathogenic bacteria- Pseudomonas and Proteus in oropharynx are noted more than PEG fed patients. ${ }^{5}$ In Japan, the use of semi-solid PEG feed is common to reduce feeding-related adverse effectsaspiration pneumonia and peristomal leakage. ${ }^{6}$ But there is no standard Indian formula for semisolid feed. The modified Indras feeding formula (table 1) differs from the standard formula in various aspects. The standard PEG feed formula is isotonic to serum, has caloric density of $1 \mathrm{kcal} / \mathrm{mL}$, lactose-free, protein content of $40 \mathrm{gm} / 1$ liter, mixture of simple and complex carbohydrates and recommended daily allowance of required nutrients. The study formula has seasonal vegetables, homemade, prepared as necessary, low cost and can be satisfactory to the care giver that the patient is sharing the same type of feed that the family is having. As in earlier studies, there were no increase in mortality or infections in intermittent

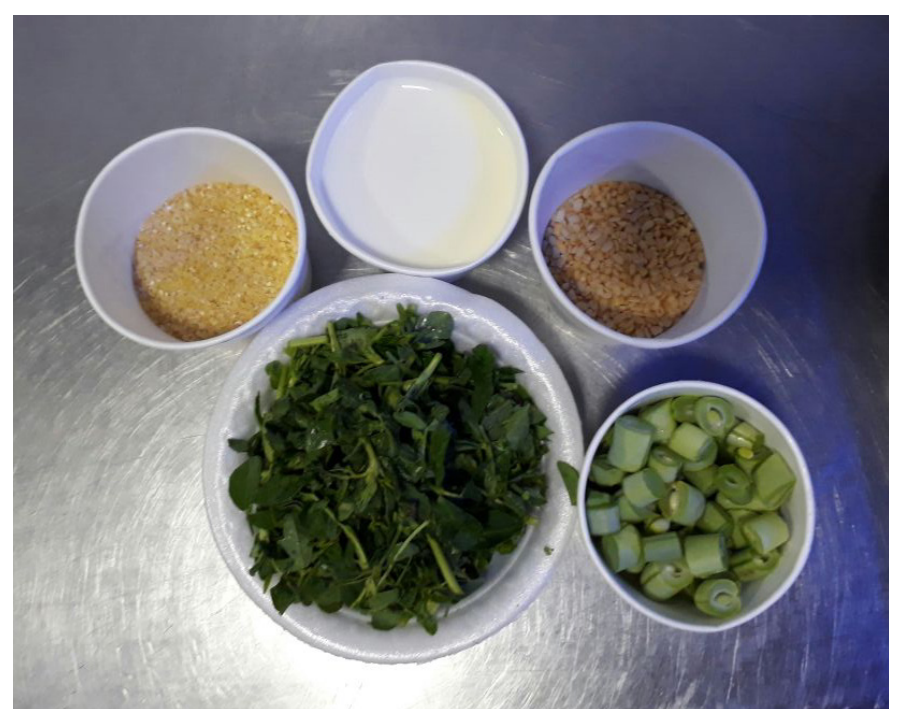

Figure 2: Showing prefeed cut vegetables.

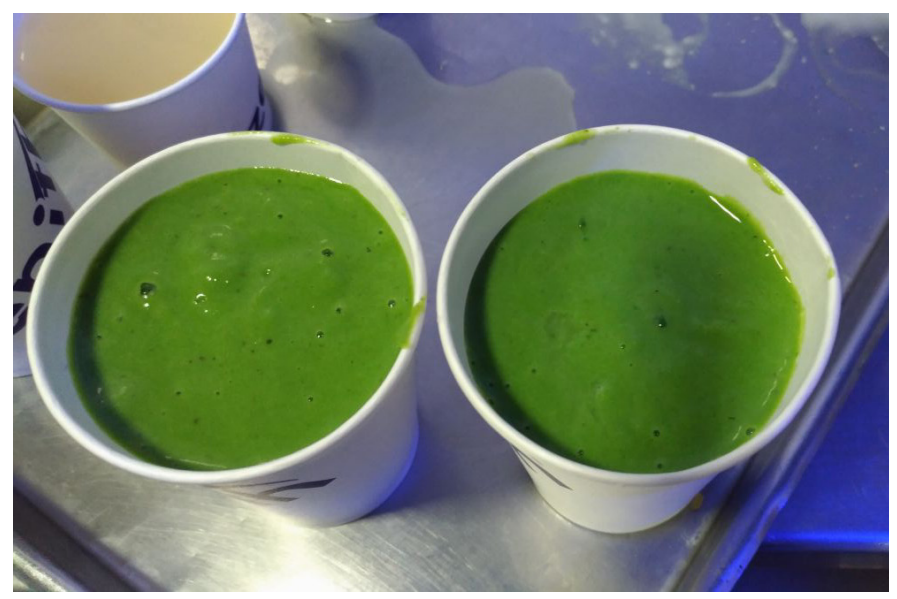

Figure 3: Showing home cooked and blenderised feed.

bolus study feeds. ${ }^{7,8}$

The average cost of the home feed for $300 \mathrm{~mL}$ is 16INR with energy content of $409 \mathrm{Kcal}$ and protein of 16 grams as compared to commercial preparation $\mathrm{XYZ}$ which costs 194 rupees with calorie content of $681 \mathrm{Kcal}$ and 49 grams protein. In home based feed- the protein content can be modified by adding more moong dal. There was a low incidence for diarrhoea in study fed patients. Diarrhoea after PEG feed could be either due to too fast feed or too cold or contamination of formula. It is recommended to proper hand wash, prepare and feed at short time, flush the tubing so that no food residue is left behind. 


\section{Conclusion}

Using home based PEG feed is safe to use and can be employed as a first-line feeding protocol

\section{References}

1. Allard JP, Keller H, Jeejeebhoy KN, et al. Decline in nutritional status is associated with prolonged length of stay in hospitalized patients admitted for 7 days or more: A prospective cohort study. ClinNutr 2016; 35:144.

2. DeLeggeMH. Percutaneous endoscopic gastrostomy. Am J Gastroenterol 2007; 102: 2620-2623.

3. Kanie J, Suzuki Y, Akatsu $\mathrm{H}$ et al. Prevention of late complications by half-solid enteral nutrients in percutaneous endoscopic gastrostomy tube feeding. Gerontology 2004; 50: 417-419.

4. Mekhail TM, Adelstein DJ, Rybicki LA, Larto MA, Saxton JP, Lavertu P. Enteral nutrition during the treatment of head and neck carcinoma: is a percutaneous endoscopic gastrostomy tube preferable to a nasogastrictube? Cancer. 2001 May 1;91(9):1785-90.

5. Segal R, Dan M, Pogoreliuk I, Leibovitz A. Pathogenic colonization of the stomach in enterally fed elderly patients: Comparing percutaneous endoscopic gastrostomy with nasogastrictube. J Am Geriatr Soc. 2006 Dec;54(12):19058.

6. Nishiwaki S, Araki H, Shirakami Y et al. Inhibition of gastroesophageal reflux by semi-solid nutrients in patients with percutaneous endoscopic gastrostomy. JPEN J Parenter Enteral Nutr 2009; 33: 513-519.

7. MacLeod JB, Lefton J, Houghton D, et al. Prospective randomized control trial of intermittent versus continuous gastric feeds for critically ill trauma patients. J Trauma 2007; 63:57.

8. EzekielWongToh Yoon, Kaori Yoneda, KazukiNishihara. Semi-solid feeds may reduce the risk of aspiration pneumonia and shorten postoperative length of stay after percutaneous endoscopic gastrostomy (PEG). Endoscopy International Open 2016; 04: E1247-E1251. 